Itinéraires Itinéraires

Littérature, textes, cultures

2013-1 | 2013

La fiction aujourd'hui

\title{
Fiction et histoire dans Les Onze de Pierre Michon
}

\section{Souad Yacoub Khlif}

\section{(2) OpenEdition}

\section{Journals}

Édition électronique

URL : http://journals.openedition.org/itineraires/821

DOI : 10.4000/itineraires.821

ISSN : 2427-920X

Éditeur

Pléiade

\section{Édition imprimée}

Date de publication : 1 octobre 2013

Pagination : 83-92

ISBN : 978-2-343-01791-4

ISSN : 2100-1340

\section{Référence électronique}

Souad Yacoub Khlif, «Fiction et histoire dans Les Onze de Pierre Michon », Itinéraires [En ligne], 2013-1 | 2013, mis en ligne le 01 octobre 2013, consulté le 10 décembre 2020. URL : http:// journals.openedition.org/itineraires/821 ; DOI : https://doi.org/10.4000/itineraires.821

Itinéraires est mis à disposition selon les termes de la licence Creative Commons Attribution - Pas d'Utilisation Commerciale - Pas de Modification 4.0 International. 


\section{Fiction et Histoire dans Les Onze de Pierre Michon}

\section{Résumé}

Les Onze de Pierre Michon s'offre à lire à la fois comme une vie imaginaire et une méditation sur l'art et l'Histoire. Ce texte entrelace, en effet, l'histoire d'un peintre, François-Élie Corentin et celle de la Révolution, à partir de la description d'un grand tableau qui serait exposé au Louvre et représentant les onze membres du Comité de salut public pendant la Terreur. Le lecteur se trouve alors projeté dans une fiction artistique où réalité et fiction se mêlent jusqu'au vertige. Michon joue de la fiction, il met en péril ses conventions et ses frontières dans une stratégie de leurre.

Mots clés : fiction, histoire, récit, biographie, biofiction

\section{Abstract}

Pierre Michon's Les Onze offers itself to be read as both an imaginary life and a meditation on art and history. In this text, the story of a painter, François-Élie Corentin, is intertwined with that of the French Revolution. This fusion takes place through the description of a large painting, which would be exhibited in the Louvre, representing the eleven members of the Comite de salut public (Committee of Public Safety) during the reign of Terror. The reader is projected into a work of artistic fiction where the real and the imaginary coalesce vertiginously. Michon plays with fiction, risking its conventions and boundaries within a strategy of fake.

Keywords: fiction, history, story, biography, biofiction

[S]i les hommes étaient faits d'étoffe indémaillable, nous ne raconterions pas d'histoires.

Pierre Michon, Les Onze, p. 22

À la parution des Onze de Pierre Michon, en avril 2009, des lecteurs courent au Louvre pour admirer le tableau de François-Élie Corentin, cette « grande toile vénitienne» de quatre mètres sur trois qu'on appelle 
Les Onze et qu'on peut voir « dans la chambre terminale du Louvre » « sous la vitre blindée de cinq pouces » ${ }^{1}$. Mais à leur grande surprise, le tableau n'y est pas, il s'agit d'une pure fiction. Comme le peintre lui-même, d'ailleurs. Les premiers critiques, interrogeant Michon sur son livre, avouent leur ignorance du tableau et s'en excusent. C'est dire combien l'illusion réaliste est parfaite et le lecteur crédule, suit les yeux fermés, puis, dépité et ravi d'être abusé de la sorte, il reprend le livre pour une « meilleure » lecture. Ces anecdotes, au-delà de leur aspect amusant peuvent nous éclairer sur la nature du traitement de la fiction chez Michon.

Ce texte ambitionne-t-il de « casser » le contrat de lecture auquel souscrit Jean-Marie Schaeffer dans Pourquoi la fiction ${ }^{2}$ ? S'agirait-il de la mise en place d'un nouveau modèle, jouant avec les limites de la fiction? C'est l'occasion d'une méditation sur la place de la fiction dans le texte et son rapport avec l'Histoire et sur tout ce jeu auquel se livre le romancier pour abolir les critères fictionnels de reconnaissance interne, au point de leurrer son lecteur.

\section{Un récit historique}

Les Onze, c'est d'abord l'histoire de la Révolution à travers celle d'un tableau «peint de la main de la Providence » (p. 44). Michon prend, en effet, pour toile de fond cet épisode majeur de l'Histoire, auquel il mêle événements et personnages fictifs. Son coup de génie est d'entrelacer subtilement éléments fictifs et histoire de la Révolution à partir de la description d'un tableau représentant une des plus terribles équipes de l'histoire de France, les onze du Comité de salut public mis en place par Robespierre pendant la Terreur et dont la liste des noms de Billaud à Saint-André se déroule aussi poétiquement qu'un chant. Tous ces membres du Comité de salut public sont des hommes des Lumières, des écrivains (à l'exception de Saint-André), « de puissantes machines à augmenter le bonheur des hommes tout en augmentant leur propre gloire » (p. 57). Michon met au centre de son récit la splendeur terrifiante de la violence de l'Histoire. Et la fiction se trouve alors ancrée dans l'Histoire, pas n'importe laquelle, «le comble de 1'Histoire » (p. 93).

Notons au passage la fascination de Michon pour l'Histoire, surtout la plus lointaine et la plus obscure. L'auteur des Vies minuscules aime s'installer dans le passé car il sait que les « choses du passé sont vertigineuses comme l'espace ${ }^{3} »$. Convaincu que « la fiction aime le passé ${ }^{4} »$, il recourt

1. Pierre Michon, Les Onze, Lagrasse, Verdier, 2009, respectivement p. 133 et p. 114. Toutes les citations successives faites dans notre étude renvoient à cette édition de l'œuvre. Nous nous limiterons donc à préciser la page entre parenthèses.

2. Jean-Marie Schaeffer, Pourquoi la fiction?, Paris, Le Seuil, 1999.

3. Pierre Michon, Vies minuscules [1984], Paris, Gallimard, coll. « Folio », 1996, p. 226.

4. Pierre Michon, Le roi vient quand il veut, propos sur la littérature, textes réunis et édités par Agnès Castiglione, Paris, Albin Michel, 2007, p. 72. 
à ces temps anciens et flous qu'évoquent les chroniques. Dans Abbés, il est allé chercher dans le Moyen Âge les personnages et les cadres qui lui permettent de transposer ses sentiments et ses impressions. Paradoxalement, le recours à l'Histoire lui semble être le meilleur moyen de " fabriquer » des fictions. Imiterait-il «l'humanité [qui] depuis qu'elle parle dit la vérité et ment, fabrique des fictions et des masques ${ }^{5} »$ ?

Le rapport des Onze à l'Histoire est, en réalité, fort complexe : la tension que Michon instaure entre dimension référentielle et fictionnelle, entre dimension réaliste et mythique est extrêmement significative. Il existe pour lui une dialectique entre le réel et l'imaginaire, l'Histoire et les histoires fictives et c'est cette dialectique qui conduit à la légende. L'écriture creuse de la sorte le vertige entre le réel et le rêve et met au jour l'essence même de l'Histoire non comme réalité, mais plutôt comme illusion. « L'Histoire chez Michon n'a d'intérêt que si elle ouvre sur la légende et les libres associations d'un imaginaire qui métamorphose l'imparfait des événements en irréel du passé ${ }^{6}$.» C'est que Michon porte sur l'Histoire un regard d'artiste, il la perçoit sur le mode de l'émotion et la passe au filtre de sa subjectivité, de sa mémoire et de son imaginaire. L'Histoire est une fiction, « résumant toutes les fictions possibles et l'idée même de fiction ${ }^{7}$ ». Tout le texte de Michon témoigne de cette intrication entre Histoire et songes personnels. Où est la part de réalité et où est la part du rêve? Est-ce de l'Histoire ou sommes-nous dans le territoire de la fiction, du rêve éveillé? Vérité et mensonge s'entrecroisent dans le paradoxe de la fiction. Comment démêler le fictif du réel? Et d'abord pourquoi faudrait-il le faire, si l'intention de l'auteur lui-même est de les confondre, de les amalgamer et de participer à la construction du mythe? Que penser d'une telle fusion? Est-ce à dire que l'Histoire, « cette falsification » et « cette reconstruction de mémoire » (p. 128) n'est qu'une page de fiction, énigmatique, presque illisible, susceptible d'appeler à une double lecture? Ou bien encore cette fiction, avec sa large part d'imagination et d'intuition, pourrait-elle paradoxalement nous révéler une part de vérité? À Daniel Morvan qui l'interroge sur la place de l'Histoire dans son texte, Michon répond : « Je mets en doute globalement toutes les représentations interprétatives de l'Histoire. » Et il ajoute : «L'Histoire, même la plus récente et la plus documentée, me paraît en fin de compte aussi opaque, mystérieuse et massivement terrible et belle que les peintures de Lascaux [...] l'Histoire c'est de la fiction, la plus haute fiction ${ }^{8}$. »

D'autre part, il est intéressant de noter que tout le récit des Onze est bâti sur une absence : « le grand tableau de la Révolution française manque ${ }^{9} »$,

5. Ibid., p. 225.

6. Michel P. Schmitt, «Les Onze de Pierre Michon », Encyclopcedia Universalis, 2009.

7. Pierre Michon, Vies minuscules, op. cit., p. 55.

8. «La horde des Onze », propos recueillis par Daniel Morvan, Ouest-France, 23 avril 2009.

9. "L'Histoire est un enchevêtrement de tragédies sans Dieu ni Grand Soir », propos recueillis par Oriane Jeancourt Galignani, Transfuge, n 30, mai 2009. 
absence que l'imagination de l'écrivain tente de combler avant de se rendre compte que ce silence est en soi éminemment significatif et en dit long sur l'Histoire et ses zones d'ombre. C'est avec ce silence que Michon travaille : ces rencontres, ces conversations que les mots fixent, cette commande que l'imagination crée et inscrit dans l'Histoire, sont tout autant réelles que la Révolution elle-même. Le tableau, inexistant dans la réalité, inexistant pour le commun des mortels, devient lui-même visible pour le lecteur par la grâce de l'écriture. Ce miracle ne peut d'ailleurs se comprendre sans se référer au mystère de l'incarnation, cher à Michon : l'écriture telle qu'il la conçoit et la pratique est parente de la résurrection des morts. Et c'est bien une des tentatives les plus audacieuses de la littérature que de vouloir non pas fixer des figures de l'Histoire, mais bien tenter de compléter l'Histoire, d'en remplir les vides : « La Révolution n'a pas produit d'œuvres d'art à la hauteur de l'événement ${ }^{10} »$, déclare Michon, et c'est le moindre droit de la fiction que de vouloir combler ce manque, réorganiser le réel et corriger ses lacunes, surtout quand cette fiction a pour elle la vérité du souffle épique.

\section{De la biofiction}

« Il était de taille médiocre, effacé, mais il retenait l'attention par son silence fiévreux, son enjouement sombre, ses manières tour à tour arrogantes et obliques - torves, on 1'a dit»(p. 11). Ainsi commence Les Onze. C'est l'occasion pour Michon de mettre en scène le destin de son peintre fictif, l'enfance puis l'ascension sociale du provincial, qui deviendra plus tard un artiste à succès. Le récit commence par dérouler sa généalogie : François-Élie Corentin, « né on le sait à Combleux en 1730 » (p. 25). D'abord l'ascendance : un grand-père maternel, ingénieur animé de ce « grand appétit souverain, magicien qui [sur la Loire] avait bâti les grandes levées toutes droites, les impeccables écluses » (p. 27). Illettré, il épouse « une fillette de vieille noblesse et de petite fortune, qui s'appelait Juliette » (p. 27). Ensuite la naissance de Suzanne, la mère du peintre, « la reine de ce monde » (p. 29) qui s'unit à un jeune homme de lettres, lequel quitte Combleux et abandonne sa femme et son fils François-Élie pour la vie parisienne où il échoue car « la chaîne des générations était trop serrée et l'étrangla » (p. 51). Nous sommes à une époque où la littérature substitue sa croyance à celle de la religion, « Dieu changeait de nid, en quelque sorte » (p. 47), et où l'écrivain croit pouvoir se penser en « puissante machine à augmenter le bonheur des hommes » (p. 48). Mais " s'il arrive que les Limousins choisissent les lettres, les lettres, elles, ne choisissent pas les Limousins » (p. 51). Aussi François Corentin de la Marche, « trop près d'un vieux maçon illettré » (p. 51), n'est-il qu'un écrivain raté, employant ses jours oisifs à une "glandouille poétique » (p. 64). Corentin fut donc « le fils d'un homme qui choisit les lettres, y sacrifia tout, et que les lettres 
brisèrent » (p. 51). Le lecteur suit l'enfant dans ses années de formation et dans son éducation : à dix ans, avec « ses boucles blondes [...] beau comme le jour, comme une fille» (p. 45), puis le joli page du monde vaporeux de Tiepolo et plus tard le « vieux crocodile» (p. 61), peintre de la Terreur. Élevé sur les bords de la Loire dans les jupons d'une mère et d'une grand-mère trop aimantes, « l'enfant vécut entre [ces] deux femmes qui le dévoraient d'amour » (p. 46). C'est l'enfant roi qui règne sur deux femmes « frileuses » et « apeurées ». On les voit tous les trois dans ce magnifique tableau où la part autobiographique est immédiatement perceptible :

L'enfant court vers la Loire, le canal, elles courent derrière lui en tenant à pleines mains leur grand panier, comme elles sont drôles, comme il s'en amuse. Comme il aime les essouffler, et comme en même temps elles l'exaspèrent - et combien aussi il est malheureux d'aimer qu'elles souffrent. Je ne vois pas le père. (p. 45-46)

Encore une fois, il s'agit de l'absence du père. Chez Michon, les pères sont toujours absents, ils sont des ombres, des spectres dont on ne parle pas. D'un texte à un autre, explicitement ou implicitement, il reconduit ce thème. Dans Vies minuscules, par exemple, premier texte de Michon, l'absence du père est centrale. L'incipit de la troisième vie, «Vies d'Eugène et de Clara » se présente comme une dédicace offerte au père, éternellement absent : « À mon père, inaccessible et caché comme un dieu, je ne saurais directement penser ${ }^{11}$ ». Ce père absent qui devient « un personnage de légende », est « le dédicataire noir des Vies minuscules ${ }^{12} »$. Il est donc bien tentant de voir en Corentin un autoportrait de l'auteur. En lui, nous retrouvons, en effet, des motifs chers à Michon : l'absence du père, les jupes féminines, l'enfance, la province. Un autoportrait en creux se dessine derrière les lignes, le personnage se présente alors comme le double fictif de Pierre Michon, comme lui, il grandit dans la présence exclusive des femmes et l'absence non regrettable du père :

je ne crois pas que [Corentin] ait souffert enfant de l'absence de son père, comme on l'a tant dit; non, le départ du père, la perte du père, ne lui fut pas une souffrance, mais un extraordinaire soulagement, une couronne inespérée (p. 63).

Et Michon d'ajouter : «celui qui n'a pas connu cela ne sait pas ce que c'est que le plaisir de vivre» (p. 63-64). Michon sait de quoi il parle, il connaît très bien «cela » pour l'avoir vécu et s'en être réjoui même. Il aime nous rappeler qu'un "père absent donne l'expérience de la plénitude $^{13} »$. À travers cette biofiction, Michon est-il en train d'« inventer et d'énoncer sa propre fable » (p. 129), comme le fera Michelet à la fin du

11. Pierre Michon, Vies minuscules, op. cit., p. 71.

12. Pierre Michon, Le roi vient quand il veut, op. cit., p. 39.

13. Ibid., p. 189. 
récit? L'image obsédante du père et du fils se déploie dans un système complexe de miroirs avec tous les jeux d'identification qu'elle suscite. C'est pourquoi dans ce tableau qui représente les Onze, Corentin « a mis son père onze fois $[\ldots]$ onze fois la revanche irréelle de son père, la défaite réelle de son père, debout » (p. 57-58). Et c'est bien une manière de traiter du meurtre du père car « toute l'histoire de la Révolution est une histoire du meurtre du père. C'est la horde des origines, comme dans Totem et Tabou de Freud : les fils, les frères (les Onze) tuent le père (le roi), et fous de culpabilité s'entretuent ${ }^{14} \gg$. Le scénario freudien prend donc place dans un récit palpitant où Michon se présente comme le « beau raconteur ${ }^{15}$ », celui qui aime à écouter ses phrases, à s'étourdir de leur rythme et de leur musicalité, bref, celui pour qui raconter est un " plaisir phénoménal ${ }^{16}$ ».

\section{« Et quand la maille se mit à filer »}

Michon s'installe avec aisance dans le territoire fictionnel, il est comme les sirènes qui « chantent à Combleux sur ce bord de Loire », « tracent des signes dans l'air » dans un " grand geste plein de signifiance » et « racontent des histoires aussi naturellement que les lavandières battent leur lessive » (p. 30). Il tient un fil et commence par dérouler la pelote. Fil finement tressé, précis et précieux à la fois. Ainsi, François-Élie Corentin grandit « comme tissé des mailles » des jupes de sa mère et de sa grand-mère, « [i]l en était encore imprégné, de leur douceur, de leur étoffe » (p. 22). Et comme la maille de leurs jupes était fortement tissée, « il fallut tailler là-dedans à pleines cisailles. Tailler, couper, trancher, faire souffrir et souffrir » (p. 60). On saisit bien ici le lien entre cette métaphore récurrente et la création. Par le truchement du champ lexical du filage - « étoffe », " mailles », " jupes 》, «tisser », " filer»- Michon installe son image de brodeur, d'artisan qui travaille pour la finesse et la beauté. Ce travail minutieux est un travail d'aiguille, patience et douceur doivent s'allier à la délicatesse et à la précision du geste. L'art de raconter est donc traité selon les mêmes modalités symboliques que l'art de filer, car «si les hommes étaient faits d'étoffe indémaillable, nous ne raconterions pas d'histoire » (p. 22).

La métaphore de l'écriture fileuse traverse toute l'écriture de Michon, de Vies minuscules à Rimbaud le fils en passant par Le Roi du bois. Tout comme Rimbaud dont les beaux vers sont « filés », Michon sait « broder le travail ténu qui vous changera en fils perpétue $1^{17} \gg$. Dans Le Roi du bois, les peintres, «avec de grands chapeaux et des barbes» font « des petits

14. « La horde des Onze », op. cit.

15. Pierre Michon, Maîtres et serviteurs, Lagrasse, Verdier, 1990, p. 127.

16. L'expression concerne l'écriture. Voir Pierre Michon, Le roi vient quand il veut, op. cit., p. 128.

17. Pierre Michon, Rimbaud le fils [1991], Paris, Gallimard, coll. « Folio », 1993, p. 32. 
travaux délicats comme quand les femmes raccommodent ${ }^{18} »$. Dans Maîtres et serviteurs, saint Martin, transfiguré dans le tableau en figure maternelle, « coupait le manteau avec des mains de couturière, suaves et exaltées, pointilleuses ${ }^{19} \gg$. Michon introduit cette métaphore dans Les Onze: «Et quand la maille se mit à filer, tout suivit, la beauté, la volonté et la confiance » (p. 22). Malgré les sirènes qui l'empêchent de parler des Onze, Michon s'abandonne à la jouissance narrative, il se laisse aller au récit, quelle que soit sa " hâte à bondir vers la fin, à commencer par la fin » (p. 23). Il sait que pour « faire tenir debout cette histoire » (p. 23), il doit commencer par le commencement. Et il sait aussi que ce mouvement ne peut exister que s'il est porté par les rêves qui «accourent comme des poussins sous leurs mères » (p. 18). Tout comme Michelet, il «n'est plus le maitre de sa fiction » car « cette fable [...] qui vient de sortir de son esprit l'enivre, l'emporte et il l'enfourche sans ambages » (p. 129).

Quant au lecteur, charmé, au sens fort du terme, il se laisse entraîner par ce flux narratif qui frappe autant par son intensité poétique et lyrique que par son opacité et son ambiguïté. La perfection de cette écriture qui opte pour la concision frappe par une très grande richesse lexicale et syntaxique et par la densité d'une prose dont l'abondance des renvois culturels demande une culture approfondie. Une écriture riche et sonore se déploie dans le cadre limité du texte court, ce qui permet à Michon de tisser une structure extrêmement serrée, de réorganiser un monde aussi complet que celui qu'un peintre inscrit sur sa toile. Et Michon nous rappelle que l'artiste est bien celui « qui veut croire de toutes ses forces, et qui arrive à croire, que l'acte par lequel on a prise sur le monde, l'acte digne de ce nom, a pour fondement et principe l'intellection pure, la magie en somme » (p. 67).

Il y a dans ce texte, une sorte de sensualité et de cruauté, une passion de l'Histoire, un goût prononcé pour l'érudition et une fascination de l'énigme. La tension de ce style, tout le système narratif mis en œuvre sont là pour témoigner de ce mystère. Dès les premières pages, le narrateur donne à entendre que son récit procédera à la manière d'un jeu de miroirs où s'efface le principe de réalité, car « chaque chose réelle existe plusieurs fois, autant de fois peut-être qu'il existe d'individus sur cette terre » (p. 126). Et c'est à un interlocuteur fictif, «Monsieur » qu'il s'adresse dans une apostrophe qui relance le récit : « Puisque vous m'en priez, Monsieur » (p. 17), «Vous les voyez, Monsieur? » (p. 43), « Je vous prie, Monsieur, d'arrêter votre attention sur ceci » (p. 39), etc. Nous relevons les caractéristiques formelles et énonciatives propres à la fictionnalité, telles qu'elles ont été déterminées par Käte Hamburger ${ }^{20}$, à savoir, la narration à la troisième personne,

18. Pierre Michon, Le Roi du bois, Lagrasse, Verdier, 1996, p. 36.

19. Pierre Michon, Maîtres et serviteurs, op. cit., p. 128.

20. Käte Hamburger, Logique des genres littéraires, Paris, Le Seuil, 1986. Cette thèse a été reprise et défendue par Dorrit Cohn dans Le Propre de la fiction, Paris, Le Seuil, coll. « Poétique », 2001. 
l'utilisation du style indirect libre qui traduit une entrée dans la conscience fictive du personnage et la perte de la signification temporelle du passé. Ainsi, Michon démonte ses propres techniques d'écriture pour montrer au lecteur que « la façon la plus sûre pour trouver la part de vérité que contient une œuvre de fiction est de ne pas être dupe de cette fiction ${ }^{21} \gg$.

\section{Un jeu d'authentification}

Mais qu'est-ce qui a fait se précipiter au musée du Louvre certains lecteurs des Onze, dans l'impatience d'admirer ce tableau de François-Élie Corentin?

On peut comprendre cette attitude en la plaçant dans une perspective qu'a développée le critique Jauss, en élaborant la notion d'horizon $d^{\prime}$ attente $^{22}$. Ce théoricien de la réception insiste sur le fait que lorsqu'une œuvre littéraire paraît, elle ne se présente jamais comme une nouveauté absolue : par tout un jeu d'annonces, de signaux, de références implicites, de caractéristiques déjà familières, son public est prédisposé à un certain mode de réception. C'est le pacte que noue tout lecteur avec un auteur ou une tradition littéraire. Il y a chez Michon une volonté affirmée de se détourner d'élaborations purement fictionnelles, de ne pas « multiplier le nombre des entités au-delà de ce qui est nécessaire ${ }^{23}$ ». « Je n'ai pas besoin d'inventer des vies, des personnages. Il y a suffisamment de gens qui sont morts et qui attendent que l'on parle d'eux ${ }^{24}$. » Avant Les Onze, Michon a consacré cinq récits à des vies de peintres réels : Vie de Joseph Roulin met en scène Van Gogh, Le Roi du bois, Claude le Lorrain et son disciple Domenico Desiderii, Maitres et serviteurs regroupe trois récits consacrés à Goya, Watteau et Lorentino, disciple de Piero della Francesca. Dans ces textes, Michon s'appuie sur des écrits existants pour élaborer la fiction et n'hésite pas à citer ses sources : les Vies de Vasari, la biographie de Caylus. Chacun de ces récits décrit les conditions et les circonstances de la naissance d'un artiste : Goya et son violent appétit d'élévation sociale, Watteau et son désir inassouvi des femmes, Desiderii et son rêve de peindre pour être prince. À travers ces récits, Michon nous livre, sous une forme à la fois fictive et réelle, des réflexions sur le champ artistique dans lequel évoluent ces artistes, sur les enjeux et les tensions qui le traversent. Tous ces éléments servent à modeler un horizon d'attente. Avec Les Onze, Michon abandonne cette perspective inaugurale, et clôt sa série picturale par un peintre imaginaire. Et c'est la première «cassure » dans le contrat de lecture. D'autre part, la fiction narrative a bien des caractères spécifiques,

21. Jean Serroy, Roman et réalité : les histoires comiques au XVII siècle, Paris, Minard, 1981, p. 53.

22. Hans Robert Jauss, Pour une esthétique de la réception, Paris, Gallimard, 1978.

23. Pierre Michon, Le roi vient quand il veut, op. cit., p. 36.

24. Ibid., p. 186. 
des « marqueurs ${ }^{25}$ » que la confrontation avec les genres non fictionnels permet de mieux percevoir.

Comment Michon s'est-il déjoué de ces «marqueurs » au point de leurrer son lecteur? De quelle magie a-t-il usé pour nous faire croire en l'existence de ce tableau?

Dans Les Onze, nous assistons à une forme de narration fictive qui implique " une feintise ludique partagée » pour reprendre la formule de Schaeffer. En effet, le narrateur ne manque pas de faire des assertions véridiques où il feint de nous raconter l'histoire vraie du peintre, "né on le sait à Combleux en $1730 »$ (p. 25, nous soulignons). Dans un acte illocutoire d'assertion et de référence à quelqu'un d'existant, il authentifie l'existence de son personnage. On adhère d'autant mieux à cette entreprise d'authentification que le narrateur nous prévient, à propos du propre portrait de ce Corentin, qu'il y aurait des faux qui circulent, et notamment celui, attribué à Vivant Denon! Michon va plus loin dans l'extrait suivant, en jouant avec la «vérité » de la biographie : les «mille biographes dont je m'inspire librement » (p. 46), et il feint même de s'interroger sur l'utilité de ces détails biographiques pour authentifier l'existence du tableau :

Je me demande, Monsieur, s'il est bien utile de vous raconter cela, ces histoires de famille et ces hautes généalogies, à quoi notre époque tient tant; s'il est besoin de remonter si loin, dans ces pâles existences qui ne sont après tout que des on-dit, des causes hypothétiques, alors que nous avons depuis deux cents ans devant nos yeux, l'existence indubitable des Onze (p. 29, nous soulignons).

Nul doute que Michon s'amuse, il se livre à un jeu de brouillage vertigineux, il manipule son lecteur, qui, ayant le sentiment réconfortant d'être en terrain familier, entre de plain-pied dans le livre en faisant confiance au texte. Quant au tableau, « le plus célèbre du monde » (p. 113) et à côté duquel « le Marat assassiné de David n'est qu'une petite toile caravagesque » (p. 133), Michon nous le décrit avec minutie jusqu'aux « reflets [qui] changent sur la vitre quand on se déplace un peu » (p. 58). Comment ne pas croire à la réalité du peintre et de son tableau après cela? Non seulement l'« état primaire » de l'acte fictionnel, comme disait Searle était complètement effacé, mais Michon choisit de « se couvrir du manteau de l'assertion ${ }^{26}$ ». À la fin du récit, il fait intervenir Michelet et l'imagine « dans le premier choc que lui causa le tableau » (p. 130), et il évoque « les douze pages de Michelet sur Les Onze dans le chapitre III du seizième livre de l'Histoire de la Révolution française » (p. 123). Il nous apprend que « Michelet déteste ce tableau autant qu'il l'admire, parce que c'est une cène truquée » (p. 131) : il marque selon lui « le retour du tyran global qui se donne pour le peuple » (p. 131).

25. Dorrit Cohn, op. cit.

26. Gérard Genette, Fiction et diction, Paris, Le Seuil, coll. « Poétique », 1991, p. 49. 
Conclusion malicieuse, la figure de Michelet vient confirmer l'authentification entretenue tout le long du récit et le narrateur tente de reconquérir par la fiction ce qui par définition ne se conquiert pas : 1'Histoire. Tout est inventé : la généalogie du peintre, la commande du tableau par trois membres du Comité révolutionnaire, le tableau lui-même, la petite notice de l'antichambre au Louvre, les douze pages de Michelet. Quand l'Histoire se fait lacunaire, n'est-ce pas le rôle de l'écriture que d'inventer, de supposer, d'imaginer, d'en emplir les creux ? Et le lecteur? Il a pu vérifier, encore une fois, que Michon a plus d'un tour dans... sa plume.

\section{Conclusion}

Les Onze se présente comme un récit original et hybride qui annonce le renouveau de la fiction et invite à s'interroger sur sa position spécifique en regard d'autres genres ou d'autres modes d'expression. Ce texte apporte, en effet, un éclairage nouveau à la fiction, notion que Michon considère comme indissociable de l'Histoire. Il reste au lecteur à démêler les fils d'un récit hybride et palpitant, génériquement inclassable, entre récit historique, mémoires, autofiction, biofiction. Cette écriture se présente comme une traversée des apparences réalistes pour atteindre une vérité légendaire et mythique. On serait là au seuil, à la limite extrême où le processus de fictionnalisation, qui permet l'accès à l'imaginaire, viendrait se ressourcer au contact même de l'Histoire et où l'on se trouve projeté sur cette rive d'hésitation faite de magie et d'enchantement. On peut affirmer, en reprenant une terminologie propre à Genette, que ce récit se range aussi bien du côté de la littérature de fiction « celle qui s'impose essentiellement par le caractère imaginaire de ses objets », que du côté de la diction, " celle qui s'impose essentiellement par ses caractéristiques formelles ${ }^{27}$ ». Michon joue de la fiction, il met en péril ses conventions et ses frontières dans une stratégie de leurre et installe son écriture dans le paradoxe. Je ne dirais pas que c'est de l'Histoire parce que ce serait une limitation, ni de la fiction parce qu'il y a plus que de la narration. Aucune des deux ne peut légitimement prétendre représenter ce texte. Il ne s'agit ni d'Histoire, ni de fiction, comme des opposés, mais d'une hybridation magique située sur la frontière indécise et mouvante du monde inventé et du monde réel. Seul l'artiste sait comment l'Histoire et la fiction collaborent. Cette dernière, nourrissant l'impossible représentation du réel historique, ne peut être saisie qu'en termes de tensions. Peut-être la modernité est-elle à ce prix.

Souad Yacoub Khlif Faculté des Lettres et des Sciences humaines de Sousse, Tunisie 